\title{
Correlations between Stroop task performance and white matter lesion measures in late-onset major depression
}

\author{
Rikke B. Dalby ${ }^{\text {a,* }}$, Jesper Frandsen ${ }^{\text {b }}$, M. Mallar Chakravarty ${ }^{\text {b,c }}$, Jamila Ahdidan ${ }^{\text {a }}$, Leif Sørensen ${ }^{\mathrm{d}}$, \\ Raben Rosenberg ${ }^{\text {, }}$, Leif Østergaard ${ }^{b}$, Poul Videbech ${ }^{\mathrm{a}}$ \\ a Centre for Psychiatric Research, Aarhus University Hospital, Risskov, Denmark \\ b Center of Functionally Integrative Neuroscience (CFIN), Aarhus University, Aarhus, Denmark \\ c PET Center, Aarhus University Hospital, Aarhus Sygehus, Aarhus, Denmark \\ d Department of Neuroradiology, Aarhus University Hospital, Aarhus Sygehus, Aarhus, Denmark
}

\section{A R T I C L E I N F O}

\section{Article history:}

Received 1 April 2011

Received in revised form 18 October 2011

Accepted 18 December 2011

\section{Keywords}

Late-onset depression

Major depression

White matter lesions

Stroop test

Psychomotor retardation

Diffusion tensor imaging

Tractography

\begin{abstract}
A B S T R A C T
Cerebral white matter lesions (WMLs) are believed to play an important role in a subset of patients with lateonset depression by affecting the white matter connectivity in circuitries essential for mood and cognition. In this study we used diffusion tensor imaging-based (DTI-based) tractography to assess white matter fiber tracts affected by deep WMLs (DWMLs) in patients with late-onset major depression and age- and gender-matched controls. Tractography outcome, illustrated as pathways affected by DWMLs, was analyzed for associations with cognitive performance on the Stroop Test (ST). The patients $(n=17)$ performed significantly worse on the ST than the controls $(n=22)$. Poor performance on the ST correlated with higher lesion load. Regression analysis showed a significant correlation between poor performance on the ST and tracts affected by DWMLs in multiple brain areas in the control group, but very sparse correlation in the patient group. Our results suggest that DWMLs play an important role in the cognitive performance of controls, whereas their influence in depressed patients is overruled by additional, state-dependent factors. Future focus on the tract-specific localization of WMLs using DTI tractography may reveal important associations between neuroconnectivity and clinical measures.
\end{abstract}

(c) 2012 Elsevier Ireland Ltd. All rights reserved.

\section{Introduction}

Depression is a heterogeneous disease with a large degree of medical co-morbidity (Evans et al., 2005). Increasing evidence suggests that cerebrovascular disease may be an important factor in the pathogenesis of a subtype of late-onset depression termed vascular depression (Alexopoulos et al., 1997a; Krishnan et al., 1997). The prevailing view is that vascular disease, in particular small-vessel disease, predisposes to depression through ischemic white matter changes, such as white matter lesions (WMLs), which are believed to disrupt or impair the white matter fiber structure in circuitries essential for mood and cognition (Alexopoulos et al., 1997a; Krishnan et al., 1997). WMLs appear as white matter hyperintensities on T2-weighted magnetic resonance imaging (MRI) of the brain and are commonly said to reflect various degrees of ischemic tissue damage ranging from mild perivascular alterations to gliosis, variable loss of fibers, and rarefaction of myelination (Fazekas et al., 1993; Pantoni and Garcia, 1997; Thomas et al., 2002; 2003). Although WMLs are a common finding in healthy

\footnotetext{
* Corresponding author at: Centre for Psychiatric Research, Aarhus University Hospital Risskov, Skovagervej 2, DK-8240 Risskov, Denmark. Tel.: + 457847 1142; fax: + 457847 1108.

E-mail address: rbdalby@dadlnet.dk (R.B. Dalby).
}

elderly individuals, imaging studies using MRI have reported an increased frequency of WMLs in depression (Videbech, 1997), especially in late-onset depression (Herrmann et al., 2008). The lesions are mainly situated in subcortical regions and their frontal white matter projections (Greenwald et al., 1998; MacFall et al., 2001; Taylor et al., 2003; Videbech et al., 2004; O'Brien et al., 2006; Sheline et al., 2008; Dalby et al., 2010a; Mueller et al., 2010), and in the basal ganglia (Videbech, 1997).

White matter abnormalities such as WMLs have been associated with impaired cognitive performance, including deficits in executive function, in both normal aging (de Groot et al., 2000; GunningDixon and Raz, 2000; Rabbitt et al., 2007; Vannorsdall et al., 2009) and depression (Goodwin, 1997; Austin et al., 2001; Herrmann et al., 2007). Severe WMLs, especially deep WMLs (DWMLs), have been suggested as a predictor of greater deficits in executive functions in late-life depression (Kohler et al., 2010).

Cognitive deficits related to late-onset depression mainly comprise reductions in processing speed and executive function (Herrmann et al., 2007), the latter covering a variety of cerebral processes, which are responsible for planning, cognitive flexibility, abstract thinking, initiating appropriate actions, and selecting relevant sensory information. A widely used neuropsychological test of executive function is the Stroop Test (ST) (Stroop, 1935). The basic principle of the test is to create 
interference between word reading and color naming, i.e. when a subject must name the color of a word which spells the name of a different color, thus creating interference between automatic and effortful information processing. In general, performance on the ST has been viewed as a sensitive, but not specific, indicator of prefrontal function, including processing speed and attentional control. The interference part of the test, in particular, is regarded as a measure of response inhibition, which is considered part of our executive control (MacLeod, 1991; Alvarez and Emory, 2006). Recent studies have correlated poor performance on the ST with an increased burden of WMLs in depression (Videbech et al., 2004; Sheline et al., 2008), suggesting that WMLs have a functional impact on neural circuitries underlying cognitive tasks, in consistency with the vascular depression hypothesis (Alexopoulos et al., 1997a; Krishnan et al., 1997) as well as the classic theories of disconnection syndromes (Catani and ffytche, 2005). In addition, the presence and severity of subcortical WMLs has been linked to psychomotor retardation (Simpson et al., 2000), which is frequently observed in late-onset depression (Alexopoulos et al., 1997b).

In spite of the increasing evidence towards WMLs playing a major role in late-onset/late-life depression, the exact anatomical and functional impact of WMLs on white matter integrity remains incompletely defined. In a recent review, Galluzzi et al. (Galluzzi et al., 2008) suggest that WMLs might affect brain function through impairment of brain plasticity and reserve. During the last decade, advances in MRI, such as diffusion tensor imaging (DTI), have facilitated the study of the microstructural integrity of brain tissues in vivo and the reconstruction of white matter trajectories, a technique known as tractography (Basser et al., 2000; Mori and van Zijl, 2002). DTI is based on the measurement of the random diffusion of water molecules in tissue (Basser et al., 1994; Pierpaoli et al., 1996), commonly described by fractional anisotropy (FA), and tractography combines the knowledge on water diffusion size and direction in each image voxel to reconstruct a fiber pathway, thus reflecting axonal connectivity (Basser et al., 2000).

The presumed mechanism whereby WMLs are associated with cognitive dysfunction involves various degrees of disruption or even disconnection of white matter circuits. We recently introduced a novel method of MRI tractography to describe the localization and impact of DWMLs on white matter structure (Dalby et al., 2010b), thereby focusing on the tract-specific localization of the lesions, which cannot be visualized with conventional MRI. We showed that DWMLs profoundly affect white matter integrity, measured by diffusion and magnetization transfer parameters, both within the lesion sites themselves and along the neuronal pathways they intersect, and do so in a similar manner in patients with late-onset major depression and controls (Dalby et al., 2010b). The aim of the present study was to use DTI tractography to examine the extent to which white matter fiber tracts affected by DWMLs are associated with cognitive deficits and psychomotor retardation in late-onset major depression compared with controls.

\section{Methods}

\subsection{Subjects}

Twenty-two patients with late-onset, first episode major depression and 22 controls, matched for age and gender and with no history of psychiatric illness, underwent whole-brain MRI. The patients were consecutively recruited from the Neuropsychiatric Clinic, Aarhus University Hospital, Risskov, Denmark, and comprised in-patients at psychiatric hospitals and out-patients from psychiatric clinics in the County of Aarhus, Denmark. Controls were recruited through advertisement in local papers. All patients met the DSM-IV (American Psychiatric Association, 2000) criteria for major depression and the ICD-10 criteria (World Health Organization, 1993) for moderate to severe depression within 4 weeks of inclusion. Late-onset was predefined as the onset of depressive symptoms after the age of 50 years. All subjects were assessed with selected parts of the SCAN structured interview (Wing et al., 1998) at study entry, including screening questions to exclude mania/hypomania (chapter 10) and psychotic symptoms (chapter 14). The interview addressed both the present state (within 4 weeks of examination) and past life, the latter to exclude any prior psychiatric history. The patients underwent a comprehensive neuropsychological examination program (results not shown), including the ST (see below). The controls were screened with the Mini-Mental State Examination (MMSE) test (Folstein et al., 1975) and also completed the ST. Depression severity was rated with the Bech-Rafaelsen Melancholia Scale (MES) (Bech, 2002) at the inclusion. Both patients and controls were thoroughly interviewed about their medical history and were screened for concurrent medical diseases and alcohol abuse by standard blood tests, including thyroid function, and they all underwent a neurological exam. All subjects were right-handed according to the Edinburgh Handedness Inventory (Oldfield, 1971), except for three patients and one control who were ambidextrous. Current medication and information on vascular risk factors, such as hypertension and smoking, were carefully recorded (Dalby et al., 2010a). Exclusion criteria for both groups were organic brain disease (e.g. former stroke, cerebral vascular malformations, epilepsy, or other known neurological diseases), former brain injury, and conventional contraindications to undergo MRI scanning. Exclusion criteria also included any lifetime psychiatric comorbidity caused by alcohol or psychoactive drugs, including substance dependency or substance abuse, defined according to the ICD-10 or DSM-IV. Written informed consent was obtained from all study subjects, and the study was approved by the regional ethics committee on research and in accordance with the Declaration of Helsinki.

\subsection{Stroop Test (ST)}

Subjects were tested with the Golden version of the ST (Golden and Freshwater, 2002), which consists of the following three parts: (I) a word part where the subject has to read aloud color names printed in black, (II) a color part where the subject has to name the ink color of printed X's, and (III) a color-word part where the subject has to name the ink color of a printed word that spells the name of a different color. The test yields three scores $(\mathrm{C}=$ color, $\mathrm{W}=$ word, and $\mathrm{CW}=$ color-word $)$ based on the number of correctly read or named items in $45 \mathrm{~s}$ in the three parts. An interference score can be calculated on the basis of the performance by the Golden formula (Golden and Freshwater, 2002):

interference score $=C W$ score-predicted $C W$ score,

where the predicted $\mathrm{CW}$ score is $=(\mathrm{W} * \mathrm{C}) /(\mathrm{W}+\mathrm{C})$. The interference score is regarded as a measure of executive functions, including selective attention and response inhibition (Goldberg and Bougakov, 2005; Alvarez and Emory, 2006). A low Golden interference score reflects a lower $\mathrm{CW}$ score than predicted, i.e. a higher degree of interference, and thus a lower task performance.

\subsection{Psychomotor retardation}

Since performance on the ST may be influenced by psychomotor slowing (Degl'Innocenti et al., 1998; Lemelin and Baruch, 1998), all subjects were additionally rated with the Widlöcher Depressive Retardation Scale (WDRS) (Widlöcher, 1983). The WDRS score refers to paucity of movements, retarded speech, paucity of thought, lack of reactivity, and difficulty of initiation.

\subsection{MRI protocol and image analysis}

MRI scans were obtained with a whole-body 3 Tesla GE Signa HDx scanner (GE Medical Systems, Milwaukee, WI, USA). The MRI protocol consisted of an axial fast spoiled gradient echo (FSPGR) 3D T1-weighted sequence $(\mathrm{TI}=750 \mathrm{~ms}$, flip angle $=14$ field of view $=240 \mathrm{~mm}$, matrix $256 * 256$, slice thickness $=1.2 \mathrm{~mm}$, no gap), an axial $\mathrm{T} 2$-weighted fluid-attenuated inversion recovery (FLAIR) sequence (TE1 $=120 \mathrm{~ms}$, $\mathrm{TR}=8650 \mathrm{~ms}, \mathrm{TI}=2250 \mathrm{~ms}$, field of view $=240 \mathrm{~mm}$, matrix $224 * 256$, slice thickness $=$ $5 \mathrm{~mm}$, gap $=1.5 \mathrm{~mm})$, an axial T2-weighted sequence $(\mathrm{TE}=112 \mathrm{~ms}, \mathrm{TR}=5400 \mathrm{~ms}$, field of view $=240 \mathrm{~mm}$, matrix $416 * 416$, slice thickness $=5 \mathrm{~mm}$, gap $=1.5 \mathrm{~mm}$ ), and an axial DTI sequence $(\mathrm{TE} 1=$ minimum, $\mathrm{TR}=12500$, flip angle $=14$, field of view $=240 \mathrm{~mm}$, matrix $128 * 128$, slice thickness $=3.5 \mathrm{~mm}$, no gap). The DTI scan was performed using double spin echo single shot echoplanar imaging with 26 gradient directions $(b=$ $1000 \mathrm{~s} / \mathrm{mm}^{2}$ ) and $6 \mathrm{~b}=0$ acquisitions. We used a birdcage head coil along with restraining foam pads to minimize the head motion.

The T1-weighted images were non-linearly transformed to match the MNI standard brain (Evans et al., 1994; Mazziotta et al., 2001). The subject-to-MNI space transformation was estimated using the ANIMAL algorithm (Collins et al., 1995; Robbins et al., 2004).

\subsection{White matter lesions}

DWMLs were identified simultaneously as hyperintensities on the T2-weighted and FLAIR images by an experienced neuroradiologist (author LS), blinded to subject status. The DWMLs were manually labeled (author RBD) on the FLAIR images using Display software (McConnell Brain Imaging Centre, MNI, McGill University, Montreal, Quebec, Canada). The labeling was performed for all subjects in random order and was completed in a single batch after the last inclusion of subjects. Initial labelings were repeated to ensure uniformity through the procedure. We used a single rater to avoid inter-rater variability. We did not test for intra-rater reliability. The labeled lesions for each subject were resampled to a binary lesion mask in native space, each voxel presenting a value of 0 (=no lesion) or 1 (=lesion). Based on previous studies reporting distinct neuropathological (Fazekas et al., 1993; Thomas et al., 2002; 2003) as well as functional differences (Kim et al., 2008) between DWMLs and periventricular WMLs (PVLs), and a stronger association between DWMLs and depression (Krishnan et al., 2006), especially 


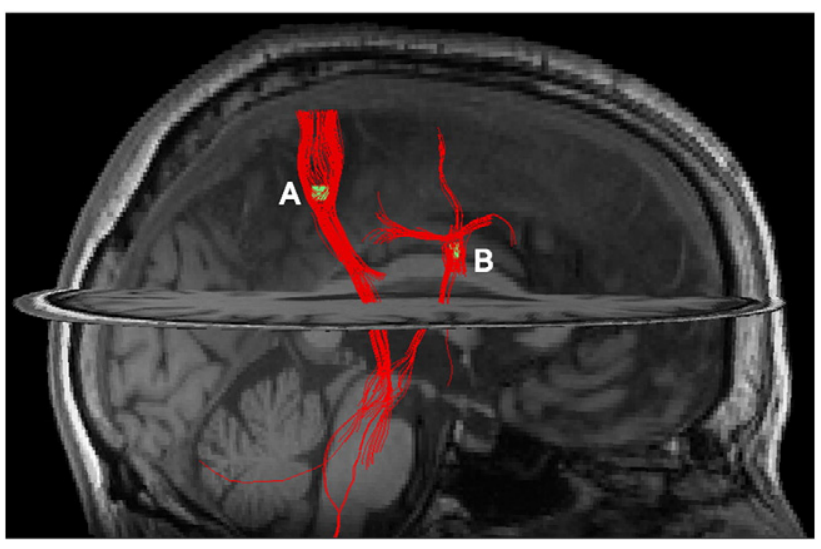

Fig. 1. Illustration of a tractography outcome (red tracts), i.e. tracts affected by DWMLs, in a subject with two DWMLs (green), marked A and B, superimposed on the subject's own T1-weighted dataset. The figure shows that one of the two lesions, marked A, situated in the posterior limb of the right internal capsule, affects far-reaching pathways, in this case extending from the cortex through the internal capsule to the brainstem, involving on its way the basal ganglia ( putamen), thalamus, and pons. DWMLs= deep white matter lesions.

late-onset depression (O'Brien et al., 1996), compared with PVLs, we omitted PVLs from assessment in order to reduce the possible neuropathological heterogeneity in the lesion pool. The lesion masks were used for measures of lesion load, i.e. volume and number of lesions, and for the tractography procedure.

\subsection{Tractography procedure}

We used in-house developed software for visualization and tractography (Moller et al., 2007); a comprehensive methodological description has recently been published (Dalby et al., 2010b). Tractography was performed in native space using the fiber assignment with continuous tracking (FACT) algorithm as described by Mori et al.
(Mori et al., 1999; Mori and van Zijl, 2002) with standard stopping criteria (FA $<0.15$ and/or angle between neighboring primary diffusion direction $>45^{\circ}$ ). Tracking was initiated from the center of every voxel in the brain. The above-mentioned individual lesion masks were then applied to the tracking algorithm to extract only the tracts intersected by one or more DWMLs for each subject. The resulting tracking mask for each individual was then saved as a binary tracking mask, each voxel assigned a value of 0 ( $=$ no tract) or 1 (= tract intersected by one or more DWMLs). Before tracking, each lesion was dilated one voxel in each direction to include the possible partial volume effects of border voxels. Fig. 1 shows an example of the tractography outcome, illustrated as pathways affected by DWMLs, from a subject with two DWMLs.

For the voxel-based analyses, all individual tracking masks were transformed to MNI standard space using the MRI-to-MNI space transformation, estimated using the T1-weighted data. These masks were subsequently blurred with a 4-mm full-widthhalf-maximum Gaussian kernel to decrease spatial noise and smooth the data.

\subsection{Statistical analyses}

Differences between patients and controls in socio-demographic and clinical data were evaluated with the Wilcoxon two-sample rank sum test (Mann-Whitney test) for continuous variables and Fisher's exact test for categorical variables. Lesion counts were analyzed with a negative binomial regression and lesion volume was modeled with a log-Gaussian regression model. Stroop scores approximated a Gaussian distribution, and differences in Stroop scores between groups, as well as correlations with psychomotor retardation, depression severity, and the number and volume of DWMLs, were evaluated by regression analyses with age and gender as covariates.

The effect of test scores on tractography outcome, i.e. the localization of fiber tracts intersected by DWMLs, was studied using voxel-wise regression analyses in FMRISTAT (Worsley et al., 2002) with age and gender as covariates. Additional adjustment for depression severity (MES score) was only carried out for the patient group, as the controls - as expected - scored 0 or very low on this scale (Table 1). Resulting $t$-maps were examined for significant changes, including summary analyses of $t$-statistic images, based on the 3D Gaussian random field theory (Worsley et al., 1996). We used the method of Cao (Cao, 1999) to identify 26-connected significant clusters at $t<-3.17$ or $t>3.17$ $(p<0.05)$. The resultant images were reviewed in Register (McConnell Brain Imaging Centre, MNI, McGill University, Montreal, Quebec, Canada) and significant clusters were anatomically located according to the DTI-based white matter atlas by Mori et al. (Wakana et al., 2004; Mori et al., 2005) and corresponding Brodmann's areas (BA) (Brodmann and Gary, 2006).

Table 1

Socio-demographic and clinical characteristics of patients with late-onset major depression and non-depressed controls.

\begin{tabular}{|c|c|c|c|c|c|c|c|c|}
\hline & \multicolumn{4}{|c|}{ Subjects } & \multicolumn{4}{|c|}{ Statistics (patients versus controls) } \\
\hline & \multicolumn{2}{|c|}{ Patients $(n=22)$} & \multicolumn{2}{|c|}{ Controls $(n=22)$} & \multicolumn{2}{|c|}{ All subjects $(n=44)$} & \multicolumn{2}{|c|}{ Subjects completing ST $(n=39)^{\mathrm{a}}$} \\
\hline & Mean & S.D. & Mean & S.D. & $\mathrm{z}$ & $p$ & $\mathrm{z}$ & $p$ \\
\hline Age (years) & 57.4 & 4.6 & 59.2 & 7.3 & 0.47 & 0.64 & 0.82 & 0.41 \\
\hline Education years & 13.5 & 2.9 & 14.8 & 2.1 & 2.29 & 0.02 & 1.58 & 0.11 \\
\hline WDRS score & 16.0 & 8.3 & 0.5 & 0.6 & -5.76 & $<0.001$ & -5.40 & $<0.001$ \\
\hline MES score & 16.5 & 5.8 & 0.3 & 0.8 & -5.93 & $<0.001$ & -5.63 & $<0.001$ \\
\hline \multirow[t]{2}{*}{ Vascular risk factor score ${ }^{\mathrm{b}}$} & 6.9 & 4.7 & 6.8 & 4.4 & 0.05 & 0.96 & & \\
\hline & $n$ & $\%$ & $n$ & $\%$ & & $p$ & & $p$ \\
\hline Gender & & & & & & 1.00 & & 0.74 \\
\hline Male & 7 & 31.8 & 7 & 31.8 & & - & & - \\
\hline Female & 15 & 68.2 & 15 & 68.2 & & - & & - \\
\hline Hypertension & 11 & 50.0 & 12 & 54.6 & & 1.00 & & 1.00 \\
\hline Smoking & & & & & & 0.02 & & 0.03 \\
\hline Current & 11 & 50.0 & 3 & 13.6 & & - & & - \\
\hline Past & 8 & 36.4 & 9 & 40.9 & & - & & - \\
\hline Never & 3 & 13.6 & 10 & 45.5 & & - & & - \\
\hline Hypercholesterolemia & 8 & 36.4 & 9 & 40.9 & & 1.00 & & 1.00 \\
\hline Diabetes & 1 & 4.6 & 1 & 4.6 & & 1.00 & & 1.00 \\
\hline \multirow[t]{2}{*}{ Cardiovascular disease $^{c}$} & 3 & 13.6 & 0 & 0.0 & & 0.23 & & 0.18 \\
\hline & \multicolumn{2}{|c|}{ Patients $(n=17)$} & \multicolumn{2}{|c|}{ Controls $(n=22)$} & & & \multicolumn{2}{|c|}{$\begin{array}{l}\text { Subjects completing } \\
\text { ST }(n=39)^{\text {a,d }}\end{array}$} \\
\hline ST scores & & & & & & & $t($ d.f. $)$ & $p$ \\
\hline Word (W) & 84.0 & 15.6 & 94.1 & 14.3 & & & $-2.03(38)$ & 0.05 \\
\hline Color (C) & 67.7 & 15.9 & 71.4 & 7.0 & & & $-0.97(38)$ & 0.34 \\
\hline Color-word (CW) & 29.1 & 11.1 & 38.5 & 9.9 & & & $-2.93(38)$ & 0.01 \\
\hline Interference & -8.3 & 11.2 & -1.9 & 9.7 & & & $-2.14(38)$ & 0.04 \\
\hline
\end{tabular}

WDRS = Widlöcher Depressive Retardation Scale; MES = Bech-Rafaelsen Melancholia Scale; ST = Stroop Test.

a Five patients did not complete ST.

b Composite vascular risk factor score comprising age, systolic blood pressure, antihypertensive treatment, diabetes, cigarette smoking, cardiovascular disease, atrial fibrillation, and left ventricular hypertrophia, as defined in the Framingham Study (Wolf et al., 1991).

c Coronary heart disease, cardiac failure, or intermittent claudication.

d Adjusted for age and gender. 


\section{Results}

The socio-demographic and clinical characteristics of all subjects are summarized in Table 1. There was no significant difference in educational level between patients and controls after excluding the five patients who did not complete the ST (see below). Patients were tested with a comprehensive neuropsychological test battery (data not shown except for the ST), whereas controls, in addition to the ST, were screened with the MMSE test $(n=22$, mean $=29.6$, S.D. $=0.49)$.

Data on the amount, volume and localization of DWMLs have been previously published (Dalby et al., 2010a) and revealed no significant difference in lesion load, i.e. number of lesions and lesion size, between patients and controls.

All 22 controls and 17 of the 22 patients completed the ST. Of the five patients who did not complete the test, four were not able to participate due to the severity of depressive symptoms (e.g. were not able to attend a neuropsychological examination) and one was precluded on linguistic grounds. The five patients who did not complete the test were excluded from ST analyses. Results from group comparisons of performance on the ST are listed in Table 1 . The patients performed significantly worse in the word and the color-word part, but not in the color part, adjusted for age and gender. Regarding interference scores, the patients had significantly lower interference scores (i.e. higher interference) than the controls, adjusted for age and gender. Regression analyses in the patient group alone revealed no significant correlation between the interference score and psychomotor retardation, measured by the WDRS ( $t=0.92$, d.f. $=16, p=0.37$ ), or depression severity, measured by MES score $(t=0.33$, d.f. $=16, p=0.75)$, adjusted for age and gender.

Regression analyses showed a correlation between the number of DWMLs and poor performance on the ST, adjusted for age and gender $(t=-2.50$, d.f. $=38, p=0.02)$. The slope parameters (i.e. change in interference score per DWML) were not significantly different between groups, with regression coefficients (model adjusted for age and gender) of $-0.12(-0.24$ to -0.01$)$ and $-0.11(-0.29$ to 0.07$)$ for patients and controls, respectively.

Regression analyses also showed a correlation between the volume of DWMLs and poor performance on the ST, adjusted for age and gender $(t=-2.47$, d.f. $=38, p=0.02)$. The slope parameters (i.e. change in interference score per $\mathrm{mm}^{3} \mathrm{DWML}$ ) were not significantly different between groups with regression coefficients (model adjusted for age and gender) of $-0.002(-0.006$ to 0.002$)$ and $-0.002(-0.004$ to -0.0002$)$ for patients and controls, respectively.
In the patient group alone, no correlation was found between psychomotor retardation, measured by the WDRS, and the number or volume of DWMLs, adjusted for age and gender $(t=-0.03$, d.f. $=21, p=0.98$ and $t=0.14$, d.f. $=21, p=0.89$, respectively).

Voxel-based analysis of the association between poor performance on the ST and white matter tracts affected by DWMLs revealed different significant clusters for the patients and controls, respectively. Figs. 2 and 3 show a representative section of the clusters significantly associated with low performance on the ST in the patient group $(n=17)$ and the control group $(n=22)$, respectively. Full view of the clusters is provided in Supplementary Fig. A (patients) and $B$ (controls). The white matter tracts affected by DWMLs significantly associated with poor test performance in the patient group comprise the white matter of A) the right superior parietal lobule adjacent to the precuneal cortex, roughly corresponding to the mesial extent of BA7 $(p=0.04)$, and B) the right middle temporal gyrus, roughly corresponding to BA21 ( $p=0.001$ ). Adjustment for depression severity (MES score) did not change this result. The clusters significantly associated with poor test performance in the control group are widespread and comprise multiple white matter structures in both hemispheres ( $p$-values ranging from $<0.001$ to just below 0.05 ), including the right superior longitudinal fasciculus (SLF), the anterior and posterior projections of the genu and splenium of the corpus callosum, as well as structures of the basal ganglia.

Finally, voxel-based analysis for the WDRS was only carried out for the patient group, as the controls - as expected - scored close to zero on this scale (Table 1 ). No significant association with tractography outcome was demonstrated for the WDRS score, adjusted for age and gen$\operatorname{der}(p \geq 0.56)$.

\section{Discussion}

The main innovation of this cross-sectional MRI study of late-onset major depression is the concomitant use of DTI tractography and FLAIR maps to identify fiber tracts affected by DWMLs and their subsequent correlation to cognitive measures. The principal finding of our study is a marked difference in the extent to which white matter fiber tracts affected by DWMLs appear to be associated with Stroop task performance in depressed patients compared with matched controls. Moreover, we showed that poor performance on the ST was correlated with increasing number and volume of DWMLs. While poor performance on the ST was associated with extensive white matter tracts affected by DWMLs in the

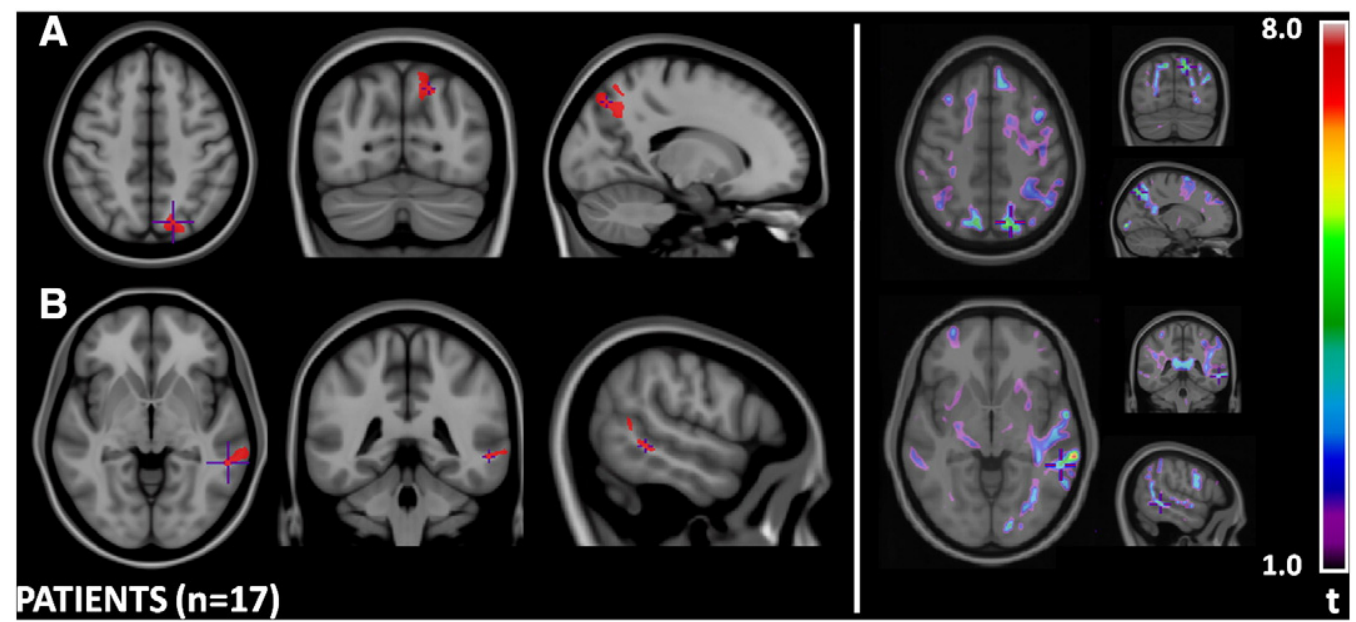

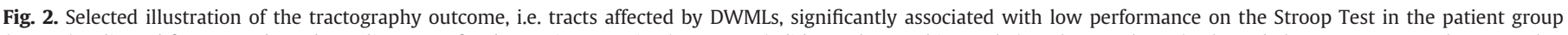

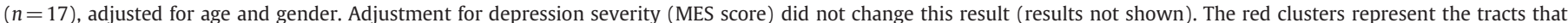

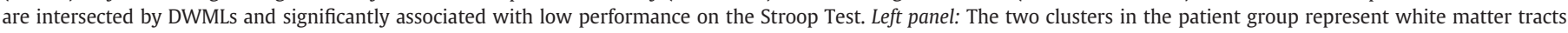

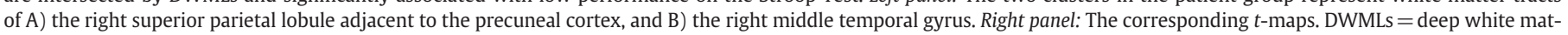
ter lesions. MES = Bech-Rafaelsen Melancholia Scale. 

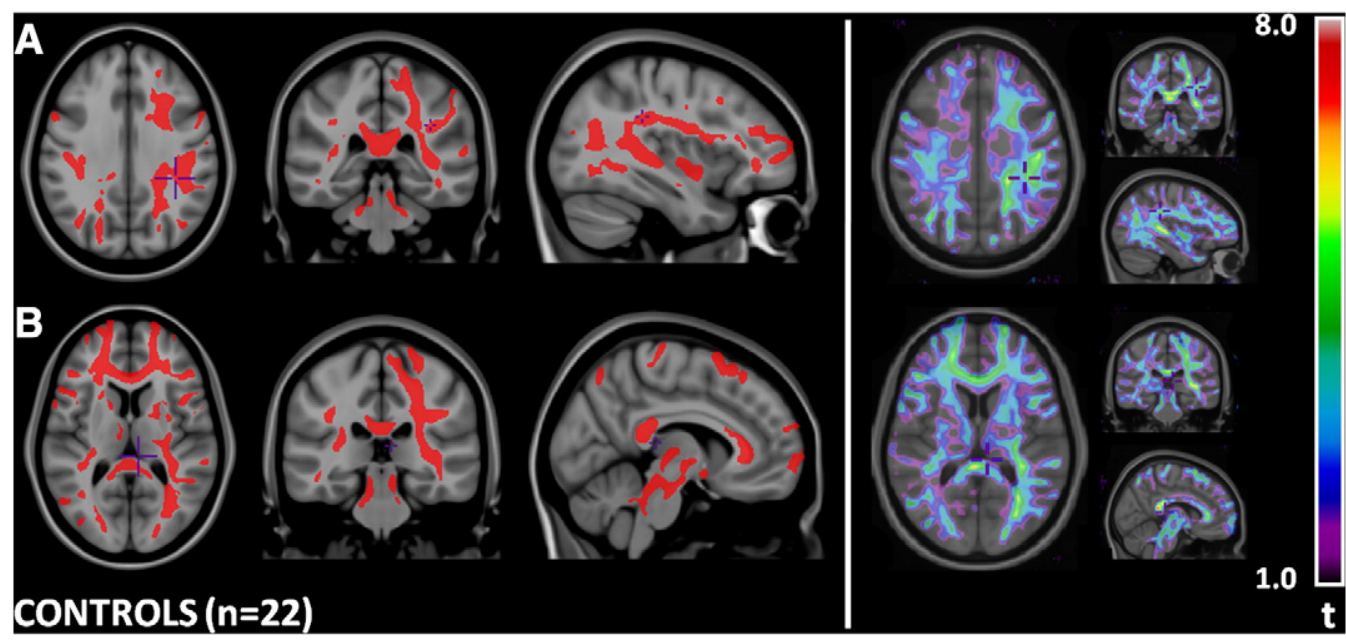

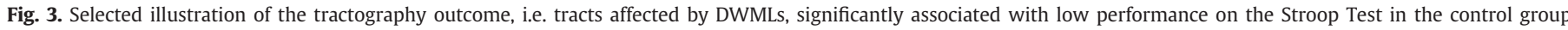

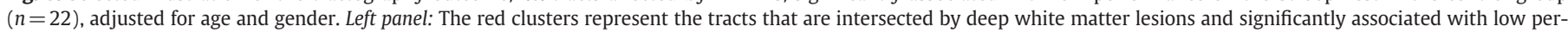

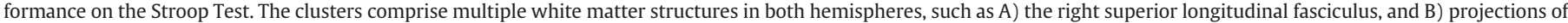
the genu and splenium of the corpus callosum, and structures of the basal ganglia. Right panel: the corresponding $t$-maps. DWMLs $=$ deep white matter lesions.

controls, the association between tractography outcome and cognitive performance in the patients was negligible, suggesting that DWMLs play an important role in the cognitive performance of controls, whereas their influence in depressed patients is counterbalanced by additional state-dependent factors.

Overall, the poorer performance on the ST in the patient group compared with the controls is in concordance with most previous findings on executive function in depression (Ottowitz et al., 2002), although controversies exist (Veiel, 1997). The association between tractography outcome, i.e. tracts affected by DWMLs, and poor cognitive performance on the ST involved different clusters in the patients compared with the controls. This was despite no significant differences in demographic variables, composite vascular risk factor score, or lesion load. While the controls showed widespread associations throughout the brain (Fig. 3, Supplementary Fig. B), the patients showed a significant association only in the right superior parietal lobule adjacent to the precuneal cortex and in the right middle temporal gyrus, the latter equivalent to the cortical BA21 (Fig. 2, Supplementary Fig. A). These areas were also represented in the controls. Functional imaging studies have shown activation of the parietal regions, including the precuneus, in a wide range of cognitive tasks, including the ST (Cabeza and Nyberg, 2000). BA21 is believed to be involved in several cognitive processes, including language (Cabeza and Nyberg, 2000). The widespread clusters in the control group (Fig. 3) comprise several important cerebral pathways, including the superior longitudinal fasciculus, the genu and splenium of the corpus callosum, and the anterior thalamic radiation. Together, these white matter structures have been attributed to a wide range of motor and cognitive functions involved in the ST, such as visual processing (Heimer, 1995), working memory (Makris et al., 2005), and selective attention (Banich, 1998). The anterior thalamic radiation in particular is a critical component of the frontal basal gangliathalamo-cortical circuits that mediate various aspects of motivation, emotional drive, planning, cognition, and motor control (Haber and Calzavara, 2009), thereby serving as an important integrative center for the complex networks underlying the ability to modulate behaviors.

The clinical aspect of cognitive performance deficits, for example, in the ST, is intriguing. Several studies have indicated that impaired executive function in late-onset and/or late-life depression is associated with poorer treatment response, suggesting that cognitive measures, including ST performance, may be a predictor of treatment resistance (Baldwin et al., 2004; Alexopoulos et al., 2005; Sheline et al., 2010; Sneed et al., 2010). The underlying neuropathological mechanisms may be explained by functional disruption of frontostriatal-limbic networks due to microstructural white matter changes such as WMLs (Alexopoulos et al., 2008; Gunning-Dixon et al., 2010), in agreement with the vascular depression hypothesis (Alexopoulos et al., 1997a; Krishnan et al., 1997). Our finding of a correlation between poor performance on the ST and higher lesion load supports this notion and is in agreement with studies in both normal aging (de Groot et al., 2000; Gunning-Dixon and Raz, 2000; Rabbitt et al., 2007; Vannorsdall et al., 2009) and depression (Videbech et al., 2004; Sheline et al., 2008).

When examining the effect of poor performance on the ST on tractography outcome, i.e. tracts affected by DWMLs, we did not find the same association patterns in patients and controls. The different association patterns, despite equal lesion load, may have several explanations. In our analyses, neither the number nor volume of DWMLs showed a difference in regression analysis between the groups. However, a similar regression coefficient (i.e. slope parameter) does not necessarily signify a similar baseline (i.e. intercept), which may be one of the explanations for the different findings in tractography outcome between patients and controls (Figs. 2 and 3), despite the similar lesion load and similar regression coefficient between groups. Cognitive performance on the ST is known to be related to, for example, atrophy of the corpus callosum (Jokinen et al., 2007) and the existence of white matter changes in prefrontal, fronto-striatal-limbic, and parietal pathways (Vendrell et al., 1995; Pujol et al., 2001; Videbech et al., 2004; Murphy et al., 2007). The extensive association pattern between poor performance on the ST and white matter fiber tracts affected by DWMLs in our control group is in agreement with a recent community study (Vannorsdall et al., 2009) in which the authors suggest the association between white matter hyperintensity burden and decreased cognitive test performance to be particularly important in older adults ( +60 years). We speculate that the sparse association pattern in the depressed patients could be due to functional abnormalities "overruling" the effect of DWMLs and thereby implying a state-dependent vulnerability. Functional imaging studies have shown increased activity during the ST in several brain regions, particularly in the anterior cingulate regions (Pardo et al., 1990; Bench et al., 1993; Carter et al., 1995; Peterson et al., 1999; Banich et al., 2000; Videbech et al., 2004). Concurrently, among the most consistent perfusion findings in depression have been reductions in the inferior frontal cortex and the adjacent cingulate cortex, temporal cortex and basal nuclei (Goodwin, 1996). In the Danish PET/depression project, Ravnkilde et al. (Ravnkilde et al., 2003) tested cognitive function and regional cerebral blood flow and concluded that disturbed cognitive functions in major depression do not relate to specific areas of the brain in the same way as normal 
cognitive functioning, suggesting that the abnormalities of brain function in major depression may be qualitative, rather than quantitative, in nature. Thus, we put forward the hypothesis that our patients in a remitted state would show the same clinical correlation pattern as our control group, in concordance with the notion that DWMLs represent a trait-dependent vulnerability.

Lastly, we detected no significant effect of psychomotor retardation (WDRS score) on performance of the ST in the patient group. This is in accordance with some previous findings (Trichard et al., 1995; Videbech et al., 2004), but not all (Degl'Innocenti et al., 1998; Lemelin and Baruch, 1998). In addition, we observed no significant association between WDRS score and tractography outcome. This is in accordance with our previous findings regarding WDRS score and localization of DWMLs (Dalby et al., 2010a). However, we cannot preclude that our study is underpowered for this specific question.

Our study comprises both strengths and weaknesses. The patient group, comprising both in-patients and out-patients, were thoroughly interviewed and examined according to inclusion and exclusion criteria and were carefully screened for medical comorbidity to minimize heterogeneity. A major strength of our study is that our patients and controls, besides being matched for age and gender, had a similar vascular risk score. Vascular risk factors have been associated with impaired to worse neuropsychological performance, e.g. in executive function, independently of the severity of WMLs (Verdelho et al., 2007), thereby emphasizing the importance of addressing the presence and distribution of vascular risk factors. Also, our two groups showed an equal amount (Dalby et al., 2010a) and tract-related distribution (Dalby et al., 2010b) of DWMLs, thereby diminishing the confounding effect of an unequal lesion load in group comparisons. Another advantage is the use of a 3 Tesla MRI scanner, which improves detection of subtle white matter changes through better spatial resolution and increased signal-to-noise ratio than seen in conventional 1.5 Tesla MRI studies.

The DTI acquisition parameters were chosen to accommodate both appropriate signal-to-noise ratio and clinically acceptable total scan time. We employed an efficient dual-gradient scheme and used a deterministic tracking method based on 26 gradient directions, which meets the requirements for most clinical purposes as the advantage in reliable determination of tensor direction after 25-30 directions is minimal (Jones, 2004). The limitations related to the use of DTI tractography are diverse, and have been scrutinized in a previous publication (Dalby et al., 2010b). At this point we cannot foretell if one or more DWMLs might result in a significant disruption to a neural pathway, as previously shown (Dalby et al., 2010b), but not enough to produce a significant correlation to behavioral outcomes as assessed by the ST. Future studies are warranted to distinguish between individual lesion severity and impact on cognitive performance.

Five of our patients did not complete the ST, four of them due to severity of depression. Several studies have shown negative correlations between depression severity and cognitive performance, possibly mediated by processing speed (Sheline et al., 2006), thus predicting a poor Stroop performance in the four excluded patients in our sample. However, controversies exist, possibly due to a ceiling effect (Videbech et al., 2004). Although regression analyses did not show any significant effect of depression severity (MES score) on Stroop interference score among the remaining 17 patients in our study, we cannot conclude that the four excluded patients did not bias the depressed group towards less severely ill individuals and thus dilute the observed significant findings. This may, however, be a strength of our study as it emphasizes the difference of findings between patients and controls despite the risk of dilution. Among potential confounders for the cognitive performance on the ST in our study are education level and medication. The correlation of education with cognitive test performance at all ages is well established, also among highly educated subjects (Lee et al., 2006). Our subjects were generally well educated (Table 1 ) with no major difference in mean education years. Our patients were not medication free, except for one who was treatment-naïve at the inclusion (Dalby et al., 2010a). Unfortunately, we were not able to restrict inclusion criteria to treatment-naïve subjects due to ethical and practical reasons. In general, psychotropic drugs are not considered important for the development of WMLs (Videbech, 1997; Herrmann et al., 2008). The effect of antidepressants on cognitive performance is a matter of debate and may depend on the specific compounds, dosage, age, and administration time (Amado-Boccara et al., 1995). However, previous reports on in-patients have suggested no significant influence of psychotropic medication on Stroop scores (Trichard et al., 1995; Ravnkilde et al., 2002).

In conclusion, we here used DTI tractography to examine correlations between white matter anatomical connectivity, cognitive performance on the ST, and psychomotor retardation in patients with late-onset major depression and matched controls. Our results suggest that DWMLs play an important role in the cognitive profile of controls, whereas their influence in depressed patients may be overruled by additional factors, e.g. changes in cerebral metabolic activity and regional blood flow. Future focus on the tract-specific localization of WMLs using DTI tractography may reveal important associations between neuroconnectivity and clinical measures, thereby providing new knowledge to aid in our understanding of cognitive deficits in neuropsychiatric disease.

Supplementary materials related to this article can be found online at doi:10.1016/j.pscychresns.2011.12.009.

\section{Financial disclosures}

None of the authors report any biomedical financial interests or potential conflicts of interest.

\section{Acknowledgment}

The authors wish to thank consultant Elisabeth Tehrani, PhD, and psychologist Barbara Ravnkilde, PhD, Neuropsychiatric Clinic, Aarhus University Hospital, Risskov, Denmark for clinical and neuropsychological assistance. For statistical advice we thank Leslie Foldager, Center for Psychiatric Research, Aarhus University Hospital, Risskov, Denmark, and Kristjana Yr Jonsdottir, CFIN, Aarhus University, Aarhus, Denmark.

The study was supported by the Danish Medical Research Council and the Research Fund for the Support of Psychiatric Research in the Central Denmark Region.

\section{References}

Alexopoulos, G.S., Meyers, B.S., Young, R.C., Campbell, S., Silbersweig, D., Charlson, M., 1997a. 'Vascular depression' hypothesis. Archives of General Psychiatry 54, 915-922.

Alexopoulos, G.S., Meyers, B.S., Young, R.C., Kakuma, T., Silbersweig, D., Charlson, M., 1997b. Clinically defined vascular depression. The American Journal of Psychiatry $154,562-565$.

Alexopoulos, G.S., Kiosses, D.N., Heo, M., Murphy, C.F., Shanmugham, B., Gunning-Dixon, F., 2005. Executive dysfunction and the course of geriatric depression. Biological Psychiatry 58, 204-210.

Alexopoulos, G.S., Murphy, C.F., Gunning-Dixon, F.M., Latoussakis, V., Kanellopoulos, D., Klimstra, S., Lim, K.O., Hoptman, M.J., 2008. Microstructural white matter abnormalities and remission of geriatric depression. The American Journal of Psychiatry $165,238-244$.

Alvarez, J.A., Emory, E., 2006. Executive function and the frontal lobes: a meta-analytic review. Neuropsychology Review 16, 17-42.

Amado-Boccara, I., Gougoulis, N., Poirier Littre, M.F., Galinowski, A., Loo, H., 1995. Effects of antidepressants on cognitive functions: a review. Neuroscience and Biobehavioral Reviews 19, 479-493.

American Psychiatric Association, 2000. Diagnostic and Statistical Manual of Mental Disorders, 4th ed. (text revision). American Psychiatric Association, Washington, DC.

Austin, M.P., Mitchell, P., Goodwin, G.M., 2001. Cognitive deficits in depression: possible implications for functional neuropathology. The British Journal of Psychiatry 178, 200-206.

Baldwin, R., Jeffries, S., Jackson, A., Sutcliffe, C., Thacker, N., Scott, M., Burns, A., 2004. Treatment response in late-onset depression: relationship to neuropsychological, neuroradiological and vascular risk factors. Psychological Medicine 34, 125-136. 
Banich, M.T., 1998. The missing link: the role of interhemispheric interaction in attentional processing. Brain and Cognition 36, 128-157.

Banich, M.T., Milham, M.P., Atchley, R., Cohen, N.J., Webb, A., Wszalek, T., Kramer, A.F., Liang, Z.P., Wright, A., Shenker, J., Magin, R., 2000. fMRI studies of Stroop tasks reveal unique roles of anterior and posterior brain systems in attentional selection. Journal of Cognitive Neuroscience 12, 988-1000.

Basser, P.J., Mattiello, J., LeBihan, D., 1994. MR diffusion tensor spectroscopy and imaging. Biophysical Journal 66, 259-267.

Basser, P.J., Pajevic, S., Pierpaoli, C., Duda, J., Aldroubi, A., 2000. In vivo fiber tractography using DT-MRI data. Magnetic Resonance in Medicine 44, 625-632.

Bech, P., 2002. The Bech-Rafaelsen Melancholia Scale (MES) in clinical trials of therapies in depressive disorders: a 20-year review of its use as outcome measure. Acta Psychiatrica Scandinavica 106, 252-264.

Bench, C.J., Frith, C.D., Grasby, P.M., Friston, K.J., Paulesu, E., Frackowiak, R.S., Dolan, R.J., 1993. Investigations of the functional anatomy of attention using the Stroop test. Neuropsychologia 31, 907-922.

Brodmann, K., Gary, L.J., 2006. Brodmann's Localization in the Cerebral Cortex: The Principles of Comparative Localisation in the Cerebral Cortex Based on Cytoarchitectonics. Springer, New York, NY.

Cabeza, R., Nyberg, L., 2000. Imaging cognition II: An empirical review of 275 PET and fMRI studies. Journal of Cognitive Neuroscience 12, 1-47.

Cao, J., 1999. The size of the connected components of excursion sets of $\mathrm{x} 2$, $\mathrm{T}$ and $\mathrm{F}$ fields. Advances in Applied Probability 31, 579-595.

Carter, C.S., Mintun, M., Cohen, J.D., 1995. Interference and facilitation effects during selective attention: an H215O PET study of Stroop task performance. Neurolmage 2, 264-272.

Catani, M., ffytche, D.H., 2005. The rises and falls of disconnection syndromes. Brain 128, 2224-2239.

Collins, D.L., Holmes, C.J., Peters, T.M., Evans, A.C., 1995. Automatic 3-D model-based neuroanatomical segmentation. Human Brain Mapping 3, 190-208.

Dalby, R.B., Chakravarty, M.M., Ahdidan, J., Sorensen, L., Frandsen, J., Jonsdottir, K.Y., Tehrani, E., Rosenberg, R., Ostergaard, L., Videbech, P., 2010a. Localization of white-matter lesions and effect of vascular risk factors in late-onset major depression. Psychological Medicine 40, 1389-1399.

Dalby, R.B., Frandsen, J., Chakravarty, M.M., Ahdidan, J., Sorensen, L., Rosenberg, R., Videbech, P., Ostergaard, L., 2010b. Depression severity is correlated to the integrity of white matter fiber tracts in late-onset major depression. Psychiatry Research: Neuroimaging $184,38-48$.

de Groot, J.C., de Leeuw, F.E., Oudkerk, M., van, G.J., Hofman, A., Jolles, J., Breteler, M.M., 2000. Cerebral white matter lesions and cognitive function: the Rotterdam Scan Study. Annals of Neurology 47, 145-151.

Degl'Innocenti, A., Agren, H., Backman, L., 1998. Executive deficits in major depression. Acta Psychiatrica Scandinavica 97, 182-188.

Evans, A.C., Collins, D.L., Mills, S.R., Brown, E.D., Kelly, R.L., Peters, T.M., 1994. 3D statistical neuroanatomical models from 305 MRI volumes. Proceedings of the 1993 IEEE Nuclear Science Symposium \& Medical Imaging Conference. IEEE, San Francisco, CA, USA, pp. 1813-1817.

Evans, D.L., Charney, D.S., Lewis, L., Golden, R.N., Gorman, J.M., Krishnan, K.R., Nemeroff, C.B., Bremner, J.D., Carney, R.M., Coyne, J.C., DeLong, M.R., Frasure-Smith, N., Glassman, A.H., Gold, P.W., Grant, I., Gwyther, L., Ironson, G., Johnson, R.L., Kanner, A.M., Katon, W.J., Kaufmann, P.G., Keefe, F.J., Ketter, T., Laughren, T.P., Leserman, J., Lyketsos, C.G., McDonald, W.M., McEwen, B.S., Miller, A.H., Musselman, D., O'Connor, C., Petitto, J.M., Pollock, B.G., Robinson, R.G., Roose, S.P., Rowland, J., Sheline, Y., Sheps, D.S., Simon, G., Spiegel, D., Stunkard, A., Sunderland, T., Tibbits Jr., P., Valvo, W.J., 2005. Mood disorders in the medically ill: scientific review and recommendations. Biological Psychiatry 58, 175-189.

Fazekas, F., Kleinert, R., Offenbacher, H., Schmidt, R., Kleinert, G., Payer, F., Radner, H., Lechner, H., 1993. Pathologic correlates of incidental MRI white matter signal hyperintensities. Neurology 43, 1683-1689.

Folstein, M.F., Folstein, S.E., McHugh, P.R., 1975. Mini-mental state. A practical method for grading the cognitive state of patients for the clinician. Journal of Psychiatric Research 12, 189-198.

Galluzzi, S., Lanni, C., Pantoni, L., Filippi, M., Frisoni, G.B., 2008. White matter lesions in the elderly: pathophysiological hypothesis on the effect on brain plasticity and reserve. Journal of the Neurological Sciences 273, 3-9.

Goldberg, E., Bougakov, D., 2005. Neuropsychologic assessment of frontal lobe dysfunction. Psychiatric Clinics of North America 28, 567-569.

Golden, C.J., Freshwater, S.M., 2002. Stroop color and word test. A Manual for Clinical and Experimental Uses. Stoelting Co., Illinois.

Goodwin, G.M., 1996. Functional imaging, affective disorder and dementia. British Medical Bulletin 52, 495-512.

Goodwin, G.M., 1997. Neuropsychological and neuroimaging evidence for the involvement of the frontal lobes in depression. Journal of Psychopharmacology 11, 115-122.

Greenwald, B.S., Kramer-Ginsberg, E., Krishnan, K.R., Ashtari, M., Auerbach, C., Patel, M., 1998. Neuroanatomic localization of magnetic resonance imaging signal hyperintensities in geriatric depression. Stroke 29, 613-617.

Gunning-Dixon, F.M., Raz, N., 2000. The cognitive correlates of white matter abnormalities in normal aging: a quantitative review. Neuropsychology 14, 224-232.

Gunning-Dixon, F.M., Walton, M., Cheng, J., Acuna, J., Klimstra, S., Zimmerman, M.E., Brickman, A.M., Hoptman, M.J., Young, R.C., Alexopoulos, G.S., 2010. MRI signal hyperintensities and treatment remission of geriatric depression. Journal of Affective Disorders 126, 395-401.

Haber, S.N., Calzavara, R., 2009. The cortico-basal ganglia integrative network: the role of the thalamus. Brain Research Bulletin 78, 69-74.

Heimer, L., 1995. The Human Brain and Spinal Cord: Functional Neuroanatomy and Dissection Guide, 2. ed. Springer-Verlag, New York.
Herrmann, L.L., Goodwin, G.M., Ebmeier, K.P., 2007. The cognitive neuropsychology of depression in the elderly. Psychological Medicine 37, 1693-1702.

Herrmann, L.L., Le, M.M., Ebmeier, K.P., 2008. White matter hyperintensities in late life depression: a systematic review. Journal of Neurology, Neurosurgery, and Psychiatry 79, 619-624.

Jokinen, H., Ryberg, C., Kalska, H., Ylikoski, R., Rostrup, E., Stegmann, M.B., Waldemar G., Madureira, S., Ferro, J.M., van Straaten, E.C., Scheltens, P., Barkhof, F., Fazekas, F., Schmidt, R., Carlucci, G., Pantoni, L., Inzitari, D., Erkinjuntti, T., 2007. Corpus callosum atrophy is associated with mental slowing and executive deficits in subjects with age-related white matter hyperintensities: the LADIS Study. Journal of Neurology, Neurosurgery, and Psychiatry 78, 491-496.

Jones, D.K., 2004. The effect of gradient sampling schemes on measures derived from diffusion tensor MRI: a Monte Carlo study. Magnetic Resonance in Medicine 51, 807-815.

Kim, K.W., MacFall, J.R., Payne, M.E., 2008. Classification of white matter lesions on magnetic resonance imaging in elderly persons. Biological Psychiatry 64, 273-280.

Kohler, S., Thomas, A.J., Lloyd, A., Barber, R., Almeida, O.P., O'Brien, J.T., 2010. White matter hyperintensities, cortisol levels, brain atrophy and continuing cognitive deficits in late-life depression. The British Journal of Psychiatry 196, 143-149.

Krishnan, K.R., Hays, J.C., Blazer, D.G., 1997. MRI-defined vascular depression. The American Journal of Psychiatry 154, 497-501.

Krishnan, M.S., O'Brien, J.T., Firbank, M.J., Pantoni, L., Carlucci, G., Erkinjuntti, T., Wallin, A., Wahlund, L.O., Scheltens, P., van Straaten, E.C., Inzitari, D., 2006. Relationship between periventricular and deep white matter lesions and depressive symptoms in older people. The LADIS Study. International Journal of Geriatric Psychiatry 21, 983-989.

Lee, S., Buring, J.E., Cook, N.R., Grodstein, F., 2006. The relation of education and income to cognitive function among professional women. Neuroepidemiology 26, 93-101.

Lemelin, S., Baruch, P., 1998. Clinical psychomotor retardation and attention in depression. Journal of Psychiatric Research 32, 81-88.

MacFall, J.R., Payne, M.E., Provenzale, J.E., Krishnan, K.R., 2001. Medial orbital frontal lesions in late-onset depression. Biological Psychiatry 49, 803-806.

MacLeod, C.M., 1991. Half a century of research on the Stroop effect: an integrative review. Psychological Bulletin 109, 163-203.

Makris, N., Kennedy, D.N., McInerney, S., Sorensen, A.G., Wang, R., Caviness Jr., V.S. Pandya, D.N., 2005. Segmentation of subcomponents within the superior longitudinal fascicle in humans: a quantitative, in vivo, DT-MRI study. Cerebral Cortex $15,854-869$.

Mazziotta, J., Toga, A., Evans, A., Fox, P., Lancaster, J., Zilles, K., Woods, R., Paus, T., Simpson, G., Pike, B., Holmes, C., Collins, L., Thompson, P., MacDonald, D., Iacoboni, M. Schormann, T., Amunts, K., Palomero-Gallagher, N., Geyer, S., Parsons, L., Narr, K. Kabani, N., Le Goualher, G., Boomsma, D., Cannon, T., Kawashima, R., Mazoyer, B., 2001. A probabilistic atlas and reference system for the human brain: International Consortium for Brain Mapping (ICBM). Philosophical Transactions of the Royal Society B: Biological Sciences 356, 1293-1322.

Moller, M., Frandsen, J., Andersen, G., Gjedde, A., Vestergaard-Poulsen, P., Ostergaard, L., 2007. Dynamic changes in corticospinal tracts after stroke detected by fibretracking. Journal of Neurology, Neurosurgery, and Psychiatry 78, 587-592.

Mori, S., van Zijl, P.C., 2002. Fiber tracking: principles and strategies - a technical review. NMR in Biomedicine 15, 468-480.

Mori, S., Crain, B.J., Chacko, V.P., van Zijl, P.C., 1999. Three-dimensional tracking of axona projections in the brain by magnetic resonance imaging. Annals of Neurology 45 , 265-269.

Mori, S., Wakana, S., Van Zijl, P.C.M., 2005. MRI Atlas of Human White Matter, 1st ed. Elsevier, Amsterdam.

Mueller, S.G., Mack, W.J., Mungas, D., Kramer, J.H., Cardenas-Nicolson, V., Lavretsky, H. Greene, M., Schuff, N., Chui, H.C., Weiner, M.W., 2010. Influences of lobar gray matter and white matter lesion load on cognition and mood. Psychiatry Research: Neuroimaging $181,90-96$.

Murphy, C.F., Gunning-Dixon, F.M., Hoptman, M.J., Lim, K.O., Ardekani, B., Shields, J.K., Hrabe, J., Kanellopoulos, D., Shanmugham, B.R. Alexopoulos, G.S. 2007. White-matte integrity predicts stroop performance in patients with geriatric depression. Biological Psychiatry 61, 1007-1010.

O'Brien, J., Desmond, P., Ames, D., Schweitzer, I., Harrigan, S., Tress, B., 1996. A magnetic resonance imaging study of white matter lesions in depression and Alzheimer's disease. The British Journal of Psychiatry 168, 477-485.

O'Brien, J.T., Firbank, M.J., Krishnan, M.S., van Straaten, E.C., van der Flier, W.M. Petrovic, K., Pantoni, L., Simoni, M., Erkinjuntti, T., Wallin, A., Wahlund, L.O. Inzitari, D., 2006. White matter hyperintensities rather than lacunar infarcts are associated with depressive symptoms in older people: the LADIS study. The American Journal of Geriatric Psychiatry 14, 834-841.

Oldfield, R.C., 1971. The assessment and analysis of handedness: the Edinburgh inventory. Neuropsychologia 9, 97-113.

Ottowitz, W.E., Dougherty, D.D., Savage, C.R., 2002. The neural network basis for abnormalities of attention and executive function in major depressive disorder: implications for application of the medical disease model to psychiatric disorders. Harvard Review of Psychiatry 10, 86-99.

Pantoni, L., Garcia, J.H., 1997. Pathogenesis of leukoaraiosis: a review. Stroke 28, 652-659.

Pardo, J.V., Pardo, P.J., Janer, K.W., Raichle, M.E., 1990. The anterior cingulate cortex mediates processing selection in the Stroop attentional conflict paradigm. Proceedings of the National Academy of Sciences of the United States of America 87, 256-259.

Peterson, B.S., Skudlarski, P., Gatenby, J.C., Zhang, H., Anderson, A.W., Gore, J.C., 1999 An fMRI study of Stroop word-color interference: evidence for cingulate subregions subserving multiple distributed attentional systems. Biological Psychiatry 45, 1237-1258. 
Pierpaoli, C., Jezzard, P., Basser, P.J., Barnett, A., Di, C.G., 1996. Diffusion tensor MR imaging of the human brain. Radiology 201, 637-648.

Pujol, J., Vendrell, P., Deus, J., Junque, C., Bello, J., Marti-Vilalta, J.L., Capdevila, A., 2001. The effect of medial frontal and posterior parietal demyelinating lesions on stroop interference. NeuroImage 13, 68-75.

Rabbitt, P., Scott, M., Lunn, M., Thacker, N., Lowe, C., Pendleton, N., Horan, M., Jackson, A., 2007. White matter lesions account for all age-related declines in speed but not in intelligence. Neuropsychology 21, 363-370.

Ravnkilde, B., Videbech, P., Clemmensen, K., Egander, A., Rasmussen, N.A., Rosenberg, R., 2002. Cognitive deficits in major depression. Scandinavian Journal of Psychology 43, 239-251.

Ravnkilde, B., Videbech, P., Clemmensen, K., Egander, A., Rasmussen, N.A., Gjedde, A., Rosenberg, R., Gade, A., 2003. The Danish PET/depression project: cognitive function and regional cerebral blood flow. Acta Psychiatrica Scandinavica 108, 32-40.

Robbins, S., Evans, A.C., Collins, D.L., Whitesides, S., 2004. Tuning and comparing spatial normalization methods. Medical Image Analysis 8, 311-323.

Sheline, Y.I., Barch, D.M., Garcia, K., Gersing, K., Pieper, C., Welsh-Bohmer, K., Steffens, D.C. Doraiswamy, P.M., 2006. Cognitive function in late life depression: relationships to depression severity, cerebrovascular risk factors and processing speed. Biological Psychiatry 60, 58-65.

Sheline, Y.I., Price, J.L., Vaishnavi, S.N., Mintun, M.A., Barch, D.M., Epstein, A.A., Wilkins, C.H., Snyder, A.Z., Couture, L., Schechtman, K., McKinstry, R.C., 2008. Regional white matter hyperintensity burden in automated segmentation distinguishes late-life depressed subjects from comparison subjects matched for vascular risk factors. The American Journal of Psychiatry 165, 524-532.

Sheline, Y.I., Pieper, C.F., Barch, D.M., Welsh-Boehmer, K., McKinstry, R.C., MacFall, J.R D'Angelo, G., Garcia, K.S., Gersing, K., Wilkins, C., Taylor, W., Steffens, D.C. Krishnan, R.R., Doraiswamy, P.M., 2010. Support for the vascular depression hypothesis in late-life depression: results of a 2-site, prospective, antidepressant treatment trial. Archives of General Psychiatry 67, 277-285.

Simpson, S., Baldwin, R.C., Jackson, A., Burns, A., Thomas, P., 2000. Is the clinical expression of late-life depression influenced by brain changes? MRI subcortical neuroanatomical correlates of depressive symptoms. International Psychogeriatrics 12, 425-434.

Sneed, J.R., Culang, M.E., Keilp, J.G., Rutherford, B.R., Devanand, D.P., Roose, S.P., 2010. Antidepressant medication and executive dysfunction: a deleterious interaction in late-life depression. The American Journal of Geriatric Psychiatry 18, 128-135.

Stroop, J.R., 1935. Studies of interference in serial verbal reactions. Journal of Experimental Psychology XVIII, 643-662.

Taylor, W.D., MacFall, J.R., Steffens, D.C., Payne, M.E., Provenzale, J.M., Krishnan, K.R. 2003. Localization of age-associated white matter hyperintensities in late-life depression. Progress in Neuro-Psychopharmacology \& Biological Psychiatry 27, 539-544.
Thomas, A.J., O'Brien, J.T., Davis, S., Ballard, C., Barber, R., Kalaria, R.N., Perry, R.H., 2002 Ischemic basis for deep white matter hyperintensities in major depression: a neuropathological study. Archives of General Psychiatry 59, 785-792.

Thomas, A.J., O'Brien, J.T., Barber, R., McMeekin, W., Perry, R., 2003. A neuropathological study of periventricular white matter hyperintensities in major depression. Journal of Affective Disorders 76, 49-54.

Trichard, C., Martinot, J.L., Alagille, M., Masure, M.C., Hardy, P., Ginestet, D., Feline, A., 1995. Time course of prefrontal lobe dysfunction in severely depressed in-patients: a longitudinal neuropsychological study. Psychological Medicine 25, 79-85.

Vannorsdall, T.D., Waldstein, S.R., Kraut, M., Pearlson, G.D., Schretlen, D.J., 2009. White matter abnormalities and cognition in a community sample. Archives of Clinical Neuropsychology 24, 209-217.

Veiel, H.O., 1997. A preliminary profile of neuropsychological deficits associated with major depression. Journal of Clinical and Experimental Neuropsychology 19, 587-603.

Vendrell, P., Junque, C., Pujol, J., Jurado, M.A., Molet, J., Grafman, J., 1995. The role of prefrontal regions in the Stroop task. Neuropsychologia 33, 341-352.

Verdelho, A., Madureira, S., Ferro, J.M., Basile, A.M., Chabriat, H., Erkinjuntti, T., Fazekas, F., Hennerici, M., O'Brien, J., Pantoni, L., Salvadori, E., Scheltens, P., Visser, M.C., Wahlund, L.O., Waldemar, G., Wallin, A., Inzitari, D., 2007. Differential impact of cerebral white matter changes, diabetes, hypertension and stroke on cognitive performance among non-disabled elderly. The LADIS study. Journal of Neurology, Neurosurgery, and Psychiatry 78, 1325-1330.

Videbech, P., 1997. MRI findings in patients with affective disorder: a meta-analysis. Acta Psychiatrica Scandinavica 96, 157-168.

Videbech, P., Ravnkilde, B., Gammelgaard, L., Egander, A., Clemmensen, K., Rasmussen, N.A., Gjedde, A., Rosenberg, R., 2004. The Danish PET/depression project: performance on Stroop's test linked to white matter lesions in the brain. Psychiatry Research: Neuroimaging 130, 117-130.

Wakana, S., Jiang, H., Nagae-Poetscher, L.M., van Zijl, P.C., Mori, S., 2004. Fiber tract-based atlas of human white matter anatomy. Radiology 230, 77-87.

Widlöcher, D.J., 1983. Psychomotor retardation: clinical, theoretical, and psychometric aspects. Psychiatric Clinics of North America 6, 27-40.

Wing, J.K., Sartorius, N., Üstün, T.B., 1998. Diagnosis and Clinical Measurement in Psychiatry. A Reference Manual for SCAN. Cambridge University Press, Cambridge.

World Health Organization, 1993. The ICD-10 classification of mental and behavioural disorders. Diagnostic criteria for research. World Health Organization, Geneva.

Worsley, K.J., Marrett, S., Neelin, P., Vandal, A.C., Friston, K.J., Evans, A.C., 1996. A unified statistical approach for determining significant signals in images of cerebral activation. Human Brain Mapping 4, 58-73.

Worsley, K.J., Liao, C.H., Aston, J., Petre, V., Duncan, G.H., Morales, F., Evans, A.C., 2002. A general statistical analysis for fMRI data. Neurolmage 15, 1-15. 